\title{
Closed-loop fault-tolerant control for uncertain nonlinear systems
}

\author{
Michel Fliess ${ }^{1}$, Cédric Join ${ }^{2}$, and Hebertt Sira-Ramírez ${ }^{3}$ \\ 1 Équipe ALIEN, INRIA Futurs \& Équipe MAX, LIX (CNRS, UMR 7161), École \\ polytechnique, 91128 Palaiseau, France. Michel.Fliess@polytechnique.fr \\ 2 Équipe ALIEN, INRIA Futurs \& CRAN (CNRS, UMR 7039), Université Henri \\ Poincaré (Nancy I), BP 239, 54506 Vandoeuvre-lés-Nancy, France. \\ cedric.join@cran.uhp-nancy.fr \\ 3 CINVESTAV-IPN, Sección de Mecatrónica, Departamento de Ingeniería \\ Eléctrica, Avenida IPN, No. 2508, Col. San Pedro Zacatenco, AP 14740, 07300 \\ México D.F., México. hsira@mail.cinvestav.mx
}

Summary. We are designing, perhaps for the first time, closed-loop fault-tolerant control for uncertain nonlinear systems. Our solution is based on a new algebraic estimation technique of the derivatives of a time signal, which

- yields good estimates of the unknown parameters and of the residuals, i.e., of the fault indicators,

- is easily implementable in real time,

- is robust with respect to a large variety of noises, without any necessity of knowing their statistical properties.

Convincing numerical simulations are provided via a popular case-study in the diagnosis community, namely the three-tank system, which may be characterized as a flat hybrid system.

Key words: Fault diagnosis, fault-tolerant control, uncertain nonlinear systems, differential algebra, algebraic estimation techniques, derivatives of a noisy time signal, three-tank system.

\section{Introduction}

We are further developing recent works on closed-loop fault detection and isolation for linear [11] and nonlinear [10, 25] systems, which may contain uncertain parameters. This important subject which is attracting more and more attention (see, e.g., $[2,3,5,19]$ and the references therein) is treated in the nonlinear case like in $[10,25]$, i.e., via differential algebra and the estimation techniques of [16]. 
Introducing on-line accommodation, or fault-tolerant control, i.e., the possibility of still controlling a nonlinear system if a fault does occur, is the main novelty of this article. We are therefore achieving in the context of diagnosis one of the fundamental aims of nonlinear control (see, e.g., [27, 30, 37] and the references therein), i.e., we are able to combine on-line parameter estimation, and closed-loop fault-tolerant control. The two main ingredients of our solution are:

- an algebraic estimation technique [15] which permits to obtain the derivatives of various orders of a noisy time signal ${ }^{1}$, and thus excellent estimates of the unknown parameters and of the residuals, i.e., of the fault indicators.

- Differential flatness (see [12, 13] and [31,32,33,35]): we all know that this standpoint is already playing a crucial rôle in many concrete and industrial control applications.

Our solution moreover is robust with respect to a large variety of noises, without any necessity of knowing their statistical properties.

Our paper is organized as follows. Section 2 is introducing the basics of the differential algebraic setting. Its content with respect to fault variables completes and supersedes [10]. Section 3 recalls the techniques for estimating the derivatives of a noisy signal. Section 4 is devoted to the three-tank system, which is perhaps the most popular case-study in the fault-diagnosis community (see, e.g., [29] and the references therein). Several simulations are illustrating our results which may be favorably compared to some recent studies on this subject (see, e.g., [22]), where only off-line diagnosis was obtained. A short conclusion indicates some prolongations.

Acknowledgement. It is an honor and a pleasure for the authors to dedicate this work to Prof. M. Zeitz for his $65^{t h}$ birthday as a tribute to his wonderful scientific achievements. At least a few words in German are in order:

gewidmet Herrn Prof. Dr.-Ing. M. Zeitz zum 65. Geburtstag.

\section{Differential algebra and nonlinear systems}

We will not recall here the basics of the approach to nonlinear systems via differential fields ${ }^{2}$, which is already well covered in the control literature (see, e.g., $[6,12,33,35]$ and the references therein).

\footnotetext{
${ }^{1}$ This method was introduced in [16] where it gave a quite straightforward solution for obtaining nonlinear state reconstructors, i.e., nonlinear state estimation, which are replacing asymptotic observers and (sub)optimal statistical filters, like the extended Kalman filters. See [9, 14] for applications in signal processing.

2 All differential rings and fields (see, e.g., [26] and [4]) are of characteristics zero and are ordinary, i.e., they are equipped with a single derivation $\frac{d}{d t}$. A differential ring $R$ is a commutative ring such that, $\forall a, b \in R$,
} 
Notation. Write $k\langle X\rangle$ (resp. $k\{X\}$ ) the differential field (resp. ring) generated by the differential field $k$ and the set $X$.

\subsection{Perturbed uncertain nonlinear systems and fault variables}

Let $k_{0}$ be a given differential ground field. Let $k=k_{0}(\boldsymbol{\Theta})$ be the the differential field extension which is generated by a finite set $\boldsymbol{\Theta}=\left(\theta_{1}, \ldots, \theta_{\alpha}\right)$ of uncertain parameters, which are assumed to be constant $^{3}$, i.e., $\dot{\theta}_{\iota}=0, \iota=1, \ldots, \alpha$. A nonlinear system is a differential field extension $K / k$, which is generated by the sets $S, \pi, \mathbf{W}$, i.e., $K=k\langle S, \pi, \mathbf{W}\rangle$, where

1. $S$ is a finite set of system variables,

2. $\pi=\left(\pi_{1}, \ldots, \pi_{r}\right)$ denotes the perturbation, or disturbance, variables,

3. $\mathbf{W}=\left(\mathbf{w}_{1}, \ldots, \mathbf{w}_{q}\right)$ denotes the fault variables.

They satisfy the following properties:

- The perturbation and fault variables do not "interact", i.e., the differential extensions $k\langle\pi\rangle / k$ and $k\langle\mathbf{W}\rangle / k$ are linearly disjoint (see, e.g., [28]).

- The fault variables are assumed to be independent, i.e., $\mathbf{W}$ is a differential transcendence basis of $k\langle\mathbf{W}\rangle / k$.

- Set $k\left\{S^{\mathrm{nom}}, \mathbf{W}^{\mathrm{nom}}\right\}=k\{S, \pi, \mathbf{W}\} /(\pi)$, where

- $(\pi) \subset k\{S, \pi, \mathbf{W}\}$ is the differential ideal generated by $\pi$,

- the nominal fault variables $S^{\text {nom }}, \mathbf{W}^{\text {nom }}$ are the canonical images of $S$, W.

Assume that the ideal $(\pi)$ is prime 4 . The nominal system is $K^{\mathrm{nom}} / k$, where $K^{\mathrm{nom}}=k\left\langle S^{\mathrm{nom}}, \mathbf{W}^{\mathrm{nom}}\right\rangle$ is the quotient field of $k\left\{S^{\mathrm{nom}}, \mathbf{W}^{\mathrm{nom}}\right\}$.

- Set $k\left\{S^{\text {pure }}\right\}=k\left\{S^{\text {nom }}, \mathbf{W}^{\text {nom }}\right\} /\left(\mathbf{W}^{\text {nom }}\right)$ where

- the differential ideal $\left(\mathbf{W}^{\text {nom }}\right) \subset k\left\{S^{\text {nom }}, \mathbf{W}^{\text {nom }}\right\}$ is generated by $\mathbf{W}^{\text {nom }}$,

- the pure system variables $S^{\text {pure }}$ are the canonical images of $S^{\text {nom }}$.

Assume that the ideal $\left(\mathbf{W}^{\text {nom }}\right)$ is prime. The pure system is $K^{\text {pure }} / k$, where $K^{\text {pure }}=k\left\langle S^{\text {pure }}\right\rangle$ is the quotient field of $k\left\{S^{\text {pure }}\right\}$.

$$
\begin{aligned}
& \frac{d}{d t}(a+b)=\dot{a}+\dot{b} \\
& \frac{d}{d t}(a b)=\dot{a} b+a \dot{b}
\end{aligned}
$$

A differential field is a differential ring which is a field. A constant is an element $c \in R$ such that $\dot{c}=0$.

${ }^{3}$ This assumption may be easily removed in our general presentation.

${ }^{4}$ An ideal $\mathfrak{I}$ of a ring $R$ is said to be prime [28] if, and only if, one of the two following equivalent conditions is verified:

- the quotient ring $R / \mathfrak{I}$ is entire, i.e., without non-trivial zero divisors,

- $\forall x, y \in R$ such that $x y \in \mathfrak{I}$, then $x \in \mathfrak{I}$ or $y \in \mathfrak{I}$.

The assumptions for $(\pi)$ and below for $\left(\mathbf{W}^{\text {nom }}\right)$ being prime are thus natural. 
A dynamics is a system where a finite subset $\mathbf{u}=\left(u_{1}, \ldots, u_{m}\right) \subset S$ of control variables has been distinguished, such that the extension $K^{\text {pure }} / k\left\langle\mathbf{u}^{\text {pure }}\right\rangle$ is differentially algebraic. The control variables verify the next two properties:

- they do not interact with the fault variables, i.e., the fields $k\langle\mathbf{u}\rangle$ and $k\langle\mathbf{W}\rangle$ are linearly disjoint over $k$.

- they are independent, i.e., the components of $\mathbf{u}$ are differentially algebraically independent over $k$.

An input-output system is a dynamics where a finite subset $\mathbf{y}=\left(y_{1}, \ldots, y_{p}\right) \subset$ $S$ of output variables has been distinguished. Only input-output systems will be considered in the sequel.

\subsection{Differential flatness}

A system $K / k$ is said to be (differentially) flat if, and only if, there exists a finite set $\mathbf{z}=\left(z_{1}, \ldots, z_{m}\right)$ of elements in the algebraic closure of $K$ such that

- its components are differentially algebraically independent over $k$,

- the algebraic closures of $K^{\text {pure }}$ and $k\left\langle\mathbf{z}^{\text {pure }}\right\rangle$ are the same.

The set $\mathbf{z}$ is called a flat output. It means that

- any pure system variable is a function of the components of the pure flat output and of their derivatives up to some finite order,

- any component of the pure flat output is a function of the pure system variables and of their derivatives up to some finite order,

- the components of the flat output are not related by any nontrivial differential relation.

The next property is well known [12, 13]:

Proposition 1. Take a flat dynamics with independent control variables, then the cardinalities of $\mathbf{z}$ and $\mathbf{u}$ are equal.

\subsection{Detectability, isolability and parity equations for fault variables}

The fault variable $\mathbf{w}_{\iota}, \iota=1, \ldots, q$, is said to be detectable if, and only if, the field extension $K^{\text {nom }} / k\left\langle\mathbf{u}^{\text {nom }}, \mathbf{W}_{\iota}^{\text {nom }}\right\rangle$, where $\mathbf{W}_{\iota}^{\text {nom }}=\mathbf{W}^{\text {nom }} \backslash\left\{\mathbf{w}_{\iota}^{\text {nom }}\right\}$, is differentially transcendental. It means that $\mathbf{w}_{\iota}$ is indeed "influencing" the output.

A subset $\mathbf{W}^{\prime}=\left(\mathbf{w}_{\iota_{1}}, \ldots, \mathbf{w}_{\iota_{q^{\prime}}}\right)$ of the set $\mathbf{W}$ of fault variables is said to be

- Differentially algebraically isolable if, and only if, the extension

$$
k\left\langle\mathbf{u}^{\text {nom }}, \mathbf{y}^{\text {nom }}, \mathbf{W}^{\prime \text { nom }}\right\rangle / k\left\langle\mathbf{u}^{\text {nom }}, \mathbf{y}^{\text {nom }}\right\rangle
$$

is differentially algebraic. It means that any component of $\mathbf{W}^{\prime \text { nom }}$ satisfies a parity differential equation, i.e., an algebraic differential equations where the coefficients belong to $k\left\langle\mathbf{u}^{\text {nom }}, \mathbf{y}^{\text {nom }}\right\rangle$. 
- Algebraically isolable if, and only if, the extension (1) is algebraic. It means that the parity differential equation is of order 0 , i.e., it is an algebraic equation with coefficients $k\left\langle\mathbf{u}^{\text {nom }}, \mathbf{y}^{\text {nom }}\right\rangle$.

- Rationally isolable if, and only if, $\mathbf{W}^{\prime \text { nom }}$ belongs to $k\left\langle\mathbf{u}^{\text {nom }}, \mathbf{y}^{\text {nom }}\right\rangle$. It means that the parity equation is a linear algebraic equation, i.e., any component of $\mathbf{W}^{\prime \text { nom }}$ may be expressed as a rational function over $k$ in the variables $\mathbf{u}^{\text {nom }}, \mathbf{y}^{\text {nom }}$ and their derivatives up to some finite order.

The next property is obvious:

Proposition 2. Rational isolability $\Rightarrow$ algebraic isolability $\Rightarrow$ differentially algebraic isolability.

When we will say for short that fault variables are isolable, it will mean that they are differentially algebraically isolable.

Proposition 3. Assume that all fault variables belonging to $\mathbf{W}^{\prime}$ are isolable, then

$$
\operatorname{card}\left(\mathbf{W}^{\prime}\right) \leq \operatorname{card}(\mathbf{y})
$$

Proof. The differential transcendence degree ${ }^{5}$ of the extension

$$
k\left\langle\mathbf{u}^{\text {nom }}, \mathbf{y}^{\text {nom }}, \mathbf{W}^{\prime \text { nom }}\right\rangle / k
$$

(resp. $k\left\langle\mathbf{u}^{\text {nom }}, \mathbf{y}^{\text {nom }}\right\rangle / k$ ) is equal to $\operatorname{card}(\mathbf{u})+\operatorname{card}\left(\mathbf{W}^{\prime}\right)$ (resp. is less than or equal to $\operatorname{card}(\mathbf{u})+\operatorname{card}(\mathbf{y}))$. The equality of those two transcendence degrees implies our result.

\subsection{Observability and identifiability}

A system variable $x$, a component of the state for instance, is said to be observable [7, 8] if, and only if, $x^{\text {pure }}$ is algebraic over $k\left\langle\mathbf{u}^{\text {pure }}, \mathbf{y}^{\text {pure }}\right\rangle$. It means in other words that $x^{\text {pure }}$ satisfies an algebraic equation with coefficients in $k\left\langle\mathbf{u}^{\text {pure }}, \mathbf{y}^{\text {pure }}\right\rangle$. It is known $[7,8]$ that under some natural and mild conditions this definition is equivalent to the classic nonlinear extension of the Kalman rank condition for observability (see, e.g., [24]).

A parameter $\theta$ is said to be algebraically (resp. rationally) identifiable $[7,8]$ if, and only if, it is algebraic over (resp. belongs to) $k\left\langle\mathbf{u}^{\text {pure }}, \mathbf{y}^{\text {pure }}\right\rangle$.

\section{Estimation of the time derivatives ${ }^{6}$}

Consider a real-valued time function $x(t)$ which is assumed to be analytic on some interval $t_{1} \leq t \leq t_{2}$. Assume for simplicity's sake that $x(t)$ is analytic around $t=0$ and introduce its truncated Taylor expansion

\footnotetext{
${ }^{5}$ See, e.g., [28] for the definition of the transcendence degree of a field extension. See [26] for its obvious generalization to differential fields.

6 See [16] and [9] for more details and related references.
} 


$$
x(t)=\sum_{\nu=0}^{N} x^{(\nu)}(0) \frac{t^{\nu}}{\nu !}+o\left(t^{N}\right)
$$

Approximate $x(t)$ in the interval $(0, \varepsilon), \varepsilon>0$, by a polynomial $x_{N}(t)=$ $\sum_{\nu=0}^{N} x^{(\nu)}(0) \frac{t^{\nu}}{\nu !}$ of degree $N$. The usual rules of symbolic calculus in Schwartz's distributions theory [34] yield

$$
x_{N}^{(N+1)}(t)=x(0) \delta^{(N)}+\dot{x}(0) \delta^{(N-1)}+\cdots+x^{(N)}(0) \delta
$$

where $\delta$ is the Dirac measure at 0 . From $t \delta=0, t \delta^{(\alpha)}=-\alpha \delta^{(\alpha-1)}, \alpha \geq 1$, we obtain the following triangular system of linear equations for determining estimated values $\left[x^{(\nu)}(0)\right]_{e}$ of the derivatives ${ }^{7} x^{(\nu)}(0)$ :

$$
\begin{array}{r}
t^{\alpha} x^{(N+1)}(t)=t^{\alpha}\left([x(0)]_{e} \delta^{(N)}+[\dot{x}(0)]_{e} \delta^{(N-1)}+\cdots+\left[x^{(N)}(0)\right]_{e} \delta\right) \\
\alpha=0, \ldots, N
\end{array}
$$

The time derivatives of $x(t)$ and the Dirac measures and its derivatives are removed by integrating with respect to time both sides of equation (2) at least $N$ times:

$$
\begin{array}{r}
\int^{(\nu)} \tau_{1}^{\alpha} x^{(N+1)}\left(\tau_{1}\right)=\int^{(\nu)} \tau_{1}^{\alpha}\left([x(0)]_{e} \delta^{(N)}+[\dot{x}(0)]_{e} \delta^{(N-1)}+\cdots+\left[x^{(N)}(0)\right]_{e} \delta\right) \\
\nu \geq N, \alpha=0, \ldots, N
\end{array}
$$

where $\int^{(\nu)}=\int_{0}^{t} \int_{0}^{\tau_{\nu-1}} \ldots \int_{0}^{\tau_{1}}$ is an iterated integral. A quite accurate value of the estimates may be obtained with a small time window $[0, t]$.

Remark 1. Those iterated integrals are moreover low pass filters ${ }^{8}$. They are attenuating highly fluctuating noises, which are usually dealt with in a statistical setting. We therefore do not need any knowledge of the statistical properties of the noises (see [14]).

\section{Application to the three-tank system}

\subsection{Process description}

The three-tank system can be conveniently represented as in [1] by:

7 Those quantities are linearly identifiable [15].

8 Those iterated integrals may be replaced by more general low pass filters, which are defined by strictly proper rational transfer functions. 


$$
\left\{\begin{aligned}
\dot{x}_{1}= & -D \mu_{1} \operatorname{sign}\left(x_{1}-x_{3}\right) \sqrt{\left|x_{1}-x_{3}\right|} \\
& +u_{1} / S+w_{1} / S \\
\dot{x}_{2}= & D \mu_{3} \operatorname{sign}\left(x_{3}-x_{2}\right) \sqrt{\left|x_{3}-x_{2}\right|} \\
& -D \mu_{2} \operatorname{sign}\left(x_{2}\right) \sqrt{\left|x_{2}\right|} \\
& +u_{2}(t) / S+w_{2} / S \\
\dot{x}_{3}= & D \mu_{1} \operatorname{sign}\left(x_{1}-x_{3}\right) \sqrt{\left|x_{1}-x_{3}\right|} \\
& -D \mu_{3} \operatorname{sign}\left(x_{3}-x_{2}\right) \sqrt{\left|x_{3}-x_{2}\right|}+w_{3} / S \\
y_{1}= & x_{1}+w_{4} \\
y_{2}= & x_{2}+w_{5} \\
y_{3}= & x_{3}+w_{6}
\end{aligned}\right.
$$

where $x_{i}, i=1,2,3$, is the liquid level in tank $i$. The control variables $u_{1}, u_{2}$ are the input flows. The actuator and/or system faults $w_{1}, w_{2}, w_{3}$ represent power losses and/or leaks; $w_{4}, w_{5}, w_{6}$ are sensor faults. The constant parameters $D$, $S$ are well known physical quantities. The viscosity coefficients $\mu_{i}, i=1,2,3$, are constant but uncertain.

The next result is an immediate consequence of proposition 3:

Proposition 4. The fault variables $w_{1}, \ldots, w_{6}$ are not simultaneously isolable.

The pure system corresponding to system (4) may be called a flat hybrid system: it is flat in each one of the four regions defined by $x_{1}>x_{3}$ or $x_{1}<x_{3}$, and $x_{2}>x_{3}$ or $x_{2}<x_{3}$. In all possible cases, $x_{1}, x_{3}$ are the components of a flat output.

\subsection{Control}

From the single outflow rate in tank 2 we may assume that system (4) is staying in the region defined by $x_{1}<x_{3}$ and/or $x_{3}<x_{2}$. We obtain the following pure open loop control, where $x_{1}^{*}=F_{1}$ and $x_{3}^{*}=F_{3}$,

$$
u_{1}^{*}=S\left(\dot{F}_{1}+D \mu_{1} \sqrt{F_{1}-F_{3}}\right)
$$

and

where

$$
u_{2}^{*}=S\left(\dot{F}_{3}-D \mu_{3} \sqrt{F_{3}-x_{2}^{*}}+D \mu_{2} \sqrt{x_{2}^{*}}\right)
$$

$$
x_{2}^{*}=F_{3}-\left(\frac{-\dot{F}_{3}+D \mu_{1} \sqrt{F_{1}-F_{3}}}{D \mu_{3}}\right)^{2}
$$

The loop is closed via a nonlinear extension (see, also, $[20,21]^{9}$ ) of the classic proportional-integral $(P I)$ controller:

$$
u_{1}=u_{1}^{*}+S D \mu_{1} \sqrt{y_{1}-y_{3}}-S D \mu_{1} \sqrt{x_{1}^{*}-x_{3}^{*}}-P_{1} S e_{1}-P_{2} S \int e_{1}
$$

\footnotetext{
${ }^{9}$ Those references also contain most useful material on the control of uncertain nonlinear systems.
} 


$$
\begin{aligned}
u_{2}= & u_{2}^{*}-S D \mu_{3} \sqrt{y_{3}-y_{2}}+S C_{2} \sqrt{y_{2}}+S D \mu_{3} \sqrt{x_{3}^{*}-x_{2}^{*}} \\
& -S D \mu_{2} \sqrt{x_{2}^{*}}-P_{3} S e_{3}-P_{4} S \int e_{3}
\end{aligned}
$$

where $e_{i}=y_{i}-F_{i}^{*}$ is the tracking error. Set for the gain coefficients

$$
P_{1}=P_{3}=2.10^{-2}, \quad P_{2}=P_{4}=2.10^{-4}
$$

\subsection{Simulation results}

\section{General principles}

The estimations of the uncertain parameters and of the residuals ${ }^{10}$ are achieved via the estimations of the first order derivatives of the output variables.

Remark 2. In order to test the robustness of our approach, we have added a zero-mean Gaussian noise of variance 0.005.

\section{Estimations of the viscosity coefficients}

The values of the known system parameters are $D=0.0144, S=0.0154$. The nominal flatness-based reference trajectories are computed via the following nominal numerical values of the viscosity coefficients

$$
\mu_{1}=\mu_{3}=0.5, \quad \mu_{2}=0.675
$$

whereas their true values are

$$
\mu_{1}^{\text {real }}=\mu_{1}(1+0.33), \quad \mu_{2}^{\text {real }}=\mu_{2}(1-0.33), \quad \mu_{3}^{\text {real }}=\mu_{3}
$$

The system behavior in the fault free case is presented figure 1. Those viscosity coefficients are algebraically identifiable:

$$
\begin{aligned}
& \mu_{1}=\frac{-\left(S \dot{y}_{1}-u_{1}\right)}{S D \sqrt{y_{1}-y_{3}}} \\
& \mu_{3}=\frac{-\left(S \dot{y}_{1}+S \dot{y}_{3}-u_{1}\right)}{S D \sqrt{y_{3}-y_{2}}} \\
& \mu_{2}=\frac{-\left(S \dot{y}_{1}+S \dot{y}_{2}+S \dot{y}_{3}-u_{1}-u_{2}\right)}{S D \sqrt{y_{2}}}
\end{aligned}
$$

Their estimations, which yield

$$
\left[\mu_{1}\right]_{c}=0.6836, \quad\left[\mu_{2}\right]_{c}=0.4339, \quad\left[\mu_{3}\right]_{c}=0.4819
$$

are presented in figure 2. After a short period of time has elapsed, those estimates become available for the implementation of our diagnosis and accommodation schemes.

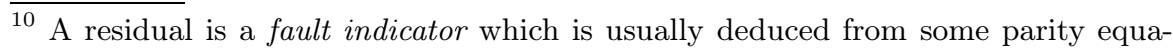
tion. Here it is obtained via the estimates of the unknown coefficients and of the derivatives of the control and output variables. 


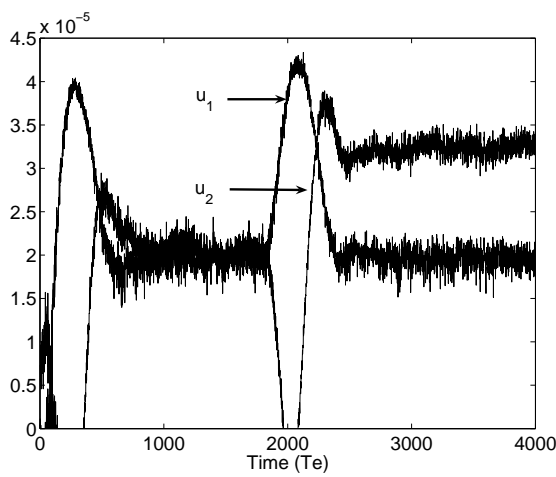

(a) Control inputs

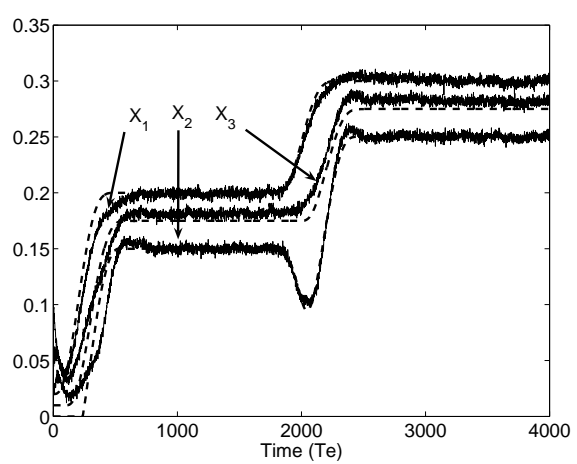

(b) Measured outputs

Fig. 1. Fault-free case

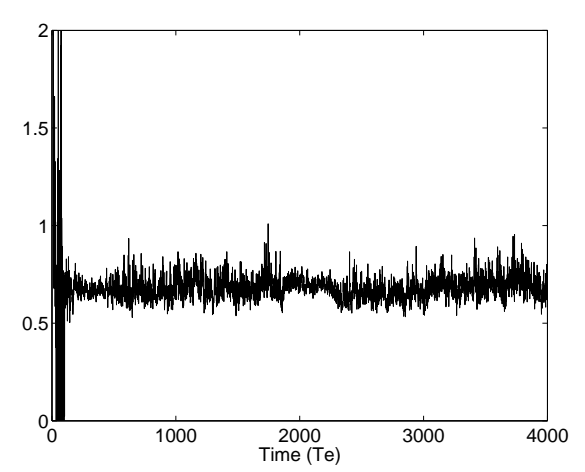

(a) $\mu_{1}$

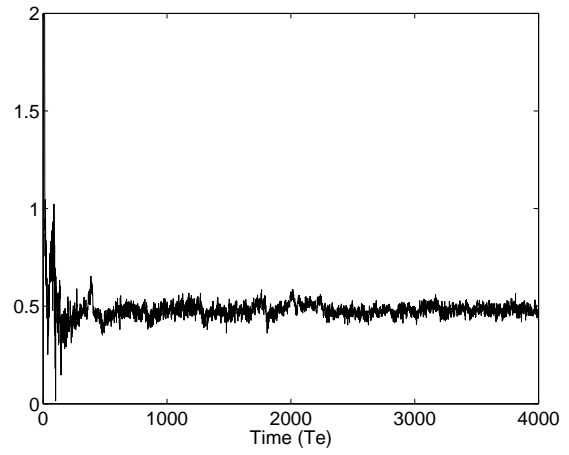

(b) $\mu_{2}$

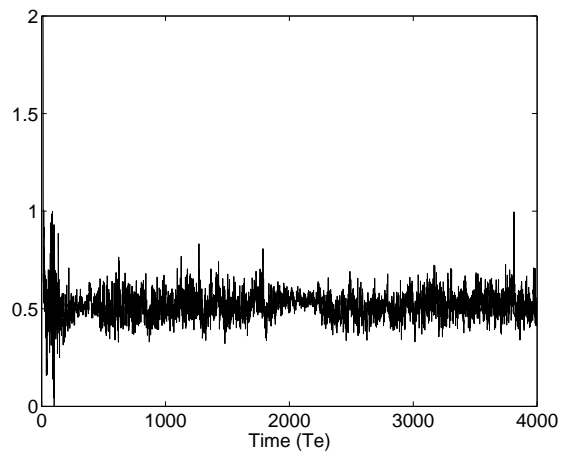

(c) $\mu_{3}$

Fig. 2. Estimations of the viscosity coefficients 


\section{Actuator and system faults}

\section{Fault diagnosis}

Assuming only the existence of the fault variables $w_{1}, w_{2}$ yields their algebraic isolability:

$$
\begin{aligned}
& w_{1}=S\left[\dot{y}_{1}+\mu_{1} D \sqrt{y_{1}-y_{3}}\right]-u_{1} \\
& w_{2}=S\left[\dot{y}_{2}-\mu_{3} D \sqrt{y_{3}-y_{2}}+\mu_{2} D \sqrt{y_{2}}\right]-u_{2}
\end{aligned}
$$

Convenient residuals $r_{1}, r_{2}$ are obtained by replacing in the above equations the viscosity coefficients $\mu_{1}, \mu_{2}, \mu_{3}$ by their estimated values $\left[\mu_{1}\right]_{c},\left[\mu_{2}\right]_{c},\left[\mu_{3}\right]_{c}$ :

$$
\begin{aligned}
& r_{1}=S\left[\dot{y}_{1}+\left[\mu_{1}\right]_{c} D \sqrt{y_{1}-y_{3}}\right]-u_{1} \\
& r_{2}=S\left[\dot{y}_{2}-\left[\mu_{3}\right]_{c} D \sqrt{y_{3}-y_{2}}+\left[\mu_{2}\right]_{c} D \sqrt{y_{2}}\right]-u_{2}
\end{aligned}
$$

\section{Fault-tolerant control}

Using the closed loop control $u_{1}$ and $u_{2}$ and the residual estimation, define a fault-tolerant control by

$$
\begin{aligned}
& u_{1}^{[\mathrm{FTC}]}=u_{1}+u_{a 1} \\
& u_{2}^{[\mathrm{FTC}]}=u_{2}+u_{a 2}
\end{aligned}
$$

where

- $u_{1}, u_{2}$ are given by formula (5),

- the additive control variables $u_{a 1}, u_{a 2}$ are defined by

$$
u_{a 1}=-r_{1}, \quad u_{a 2}=-r_{2}
$$

The simulations are realized by assuming a detection delay $T_{d i}$ of the fault variable $w_{i}$.

\section{Simulation comments}

Figures 3-(c)-(d), 4-(c)-(d) and 5-(c)-(d) indicate an excellent fault diagnosis for the following three classic cases (see, e.g., [17]):

1. $w_{1}=-0.5 u_{1}, w_{2}=-0.5 u_{2}$, for $t>1000 T_{e}$, where $T_{e}$ is the sampling period (figures 3 ),

2. $w_{1}=-0.5 u_{1}, w_{2}=-0.5 u_{2}$ for $t>2000 T_{e}$ (figures 4 ),

3. $w_{1}=\left(-0.5-\frac{t}{16000 T_{e}}\right) u_{1}, w_{2}=\left(-0.5-\frac{t}{16000 T_{e}}\right) u_{2}$, for $t>1000 T_{e}$ (figures $5)$.

The behavior for the residuals changes at time $t=500 T_{e}$. This is due to the fact that the nominal value of $\mu_{i}$ is being used for $t<500 T_{e}$. The interest of the fault-tolerant control is demonstrated in figures 3,4 and 5 . Note that the simulations were realized with a delay of $T_{d i}=100 \mathrm{Te}$ for the fault-tolerant control.

In figure 6 the system is corrupted by two major faults variables, where $w_{1}=-0.9 u_{1}, w_{2}=-0.9 u_{2}$ for $t>1000 T_{e}$. The fault-tolerant control is then saturating the actuator. The output references cannot be reached. 


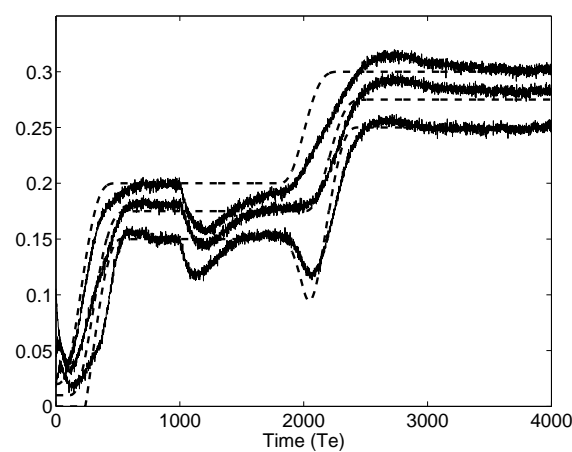

(a) Without fault-tolerant control

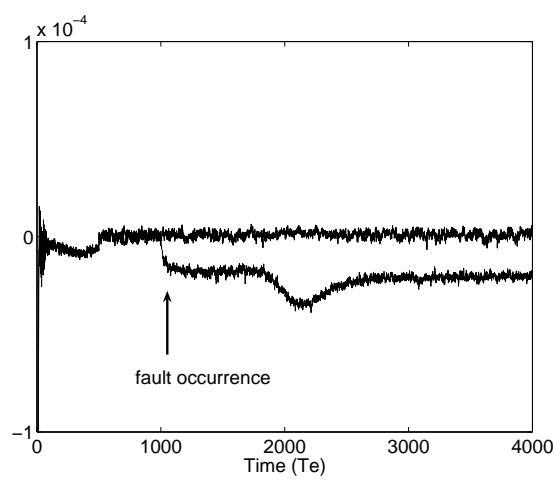

(c) $r_{1}$

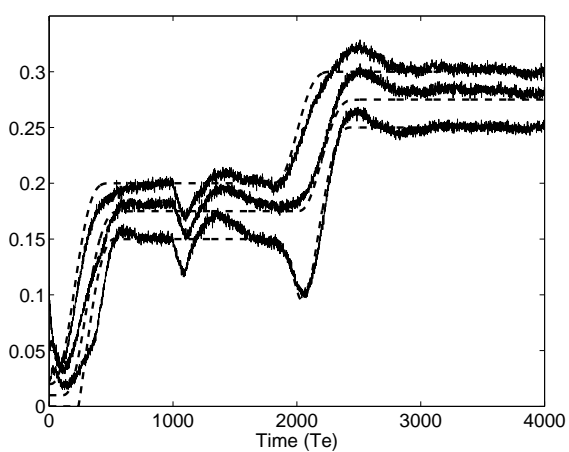

(b) With fault-tolerant control

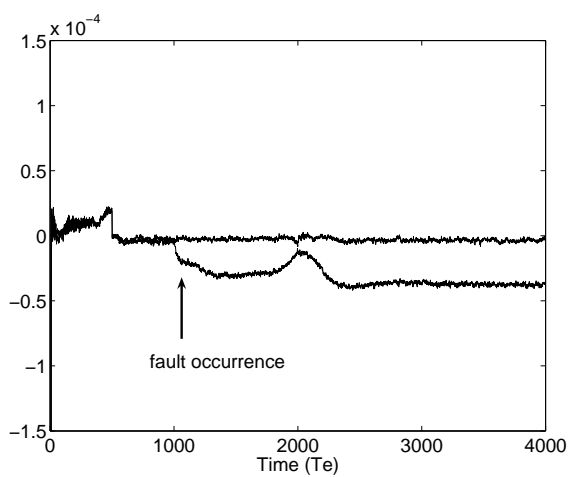

(d) $r_{2}$

Fig. 3. Fault occurrence in steady mode

\section{Combination of system and sensor faults}

Fault diagnosis

We associate here the leak $w_{2}$ and the sensor fault $w_{4}$, which is algebraically isolable:

$$
w_{4}=y_{1}-y_{3}-\left(\frac{\dot{y}_{3}+\mu_{3} D\left(y_{3}-y_{2}\right) \sqrt{y_{3}-y_{2}}}{\mu_{1} D}\right)^{2}
$$

It yields in the same way as before the residual

$$
r_{4}=y_{1}-y_{3}-\left(\frac{\dot{y}_{3}+\left[\mu_{3}\right]_{c} D\left(y_{3}-y_{2}\right) \sqrt{y_{3}-y_{2}}}{\left[\mu_{1}\right]_{c} D}\right)^{2}
$$

\section{Fault-tolerant control}

The leak $w_{2}$ is accommodated as in section 4.3. For the sensor fault $w_{4}$, accommodation is most simply achieved by subtracting $r_{4}$ from the measurement $y_{1}$ when closing the loop (5). 


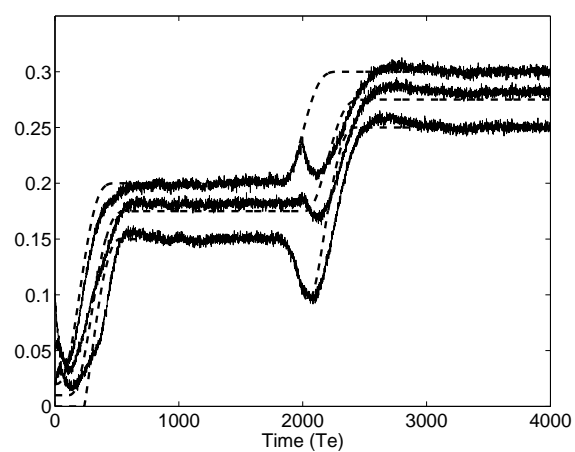

(a) Without fault-tolerant control

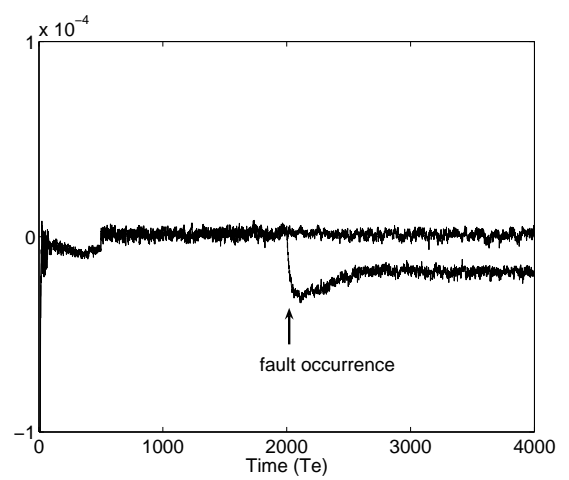

(c) $r_{1}$

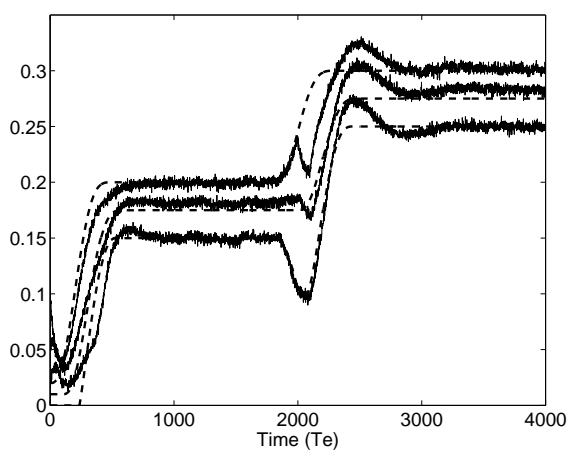

(b) With fault-tolerant control

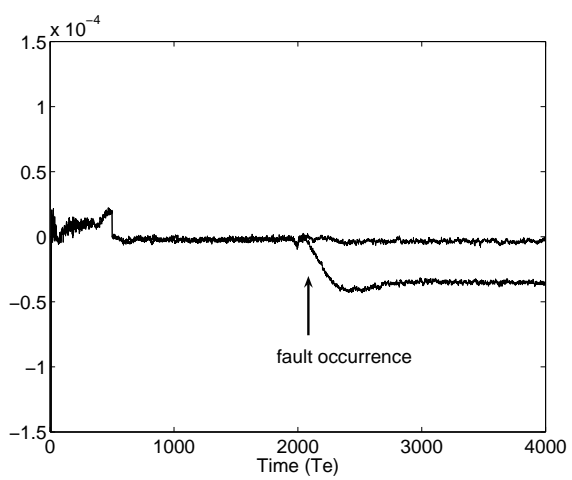

(d) $r_{2}$

Fig. 4. Fault occurrence in dynamical mode

\section{Simulation comments}

Figures 7-(c)-(d) (resp. 7-(a)-(b)) show an excellent fault diagnosis (resp. accommodation) for $w_{2}(t)=-0.3\left[\mu_{2}\right]_{c} D \sqrt{x_{2}}$, for $t>1000 T_{e}$, and $w_{4}(t)=0.02$, for $t>2500 T_{e}$.

Remark 3. Figures 8-(a) and 8-(b), when compared to figures 1-(b) and 7-(b), show quite better performances of the feedback loop (5) when the nominal values of the viscosity coefficients are replaced by the estimated ones.

\section{Conclusion}

This communication should be viewed as a first draft of a full paper which will comprise also state estimation $[16,36]$ and many more examples. Those simple 


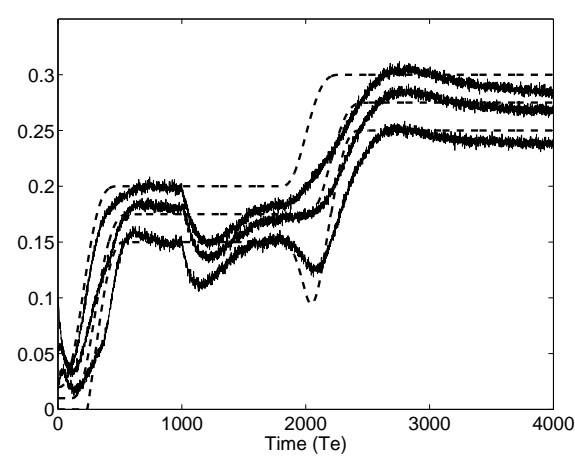

(a) Without fault-tolerant control

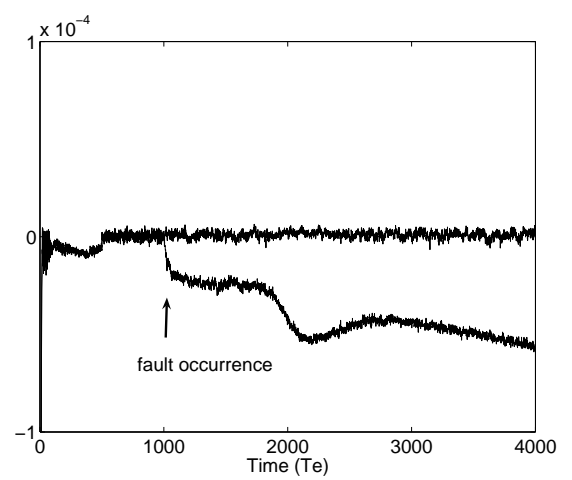

(c) $r_{1}$

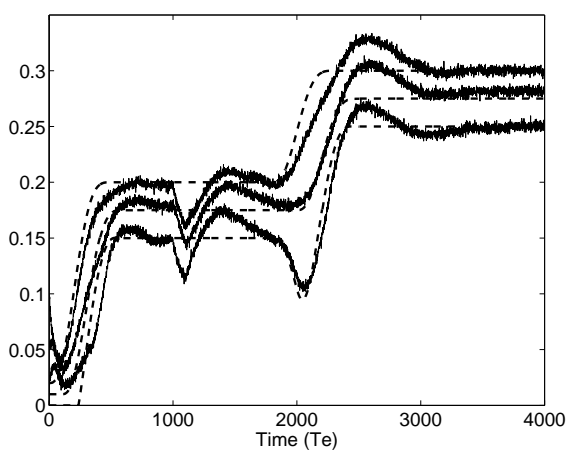

(b) With fault-tolerant control

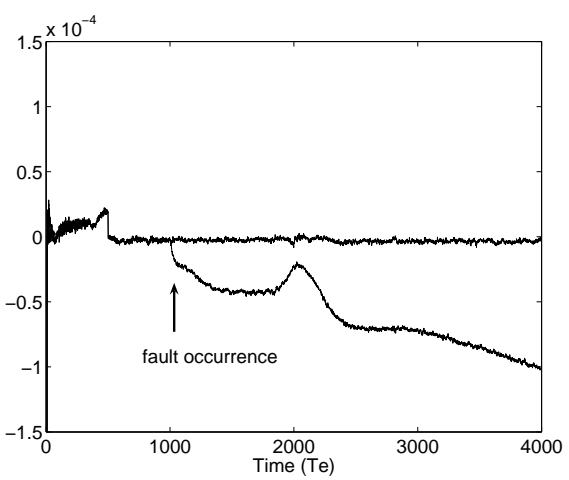

(d) $r_{2}$

Fig. 5. Fault of type 3

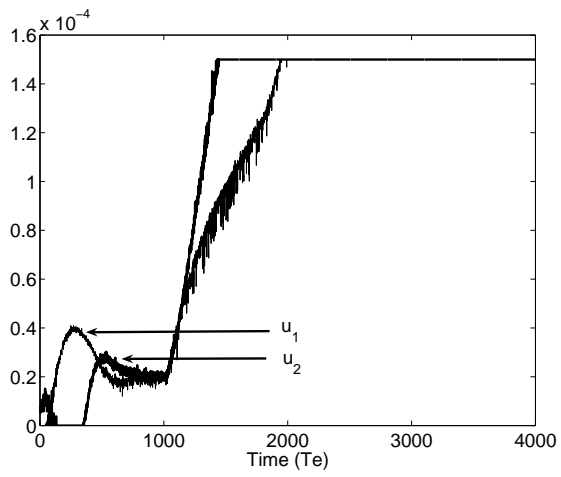

(a) Control inputs

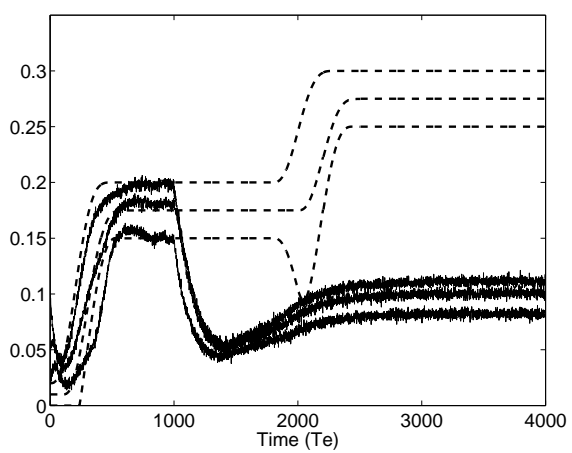

(b) Measured outputs

Fig. 6. Actuator saturations 


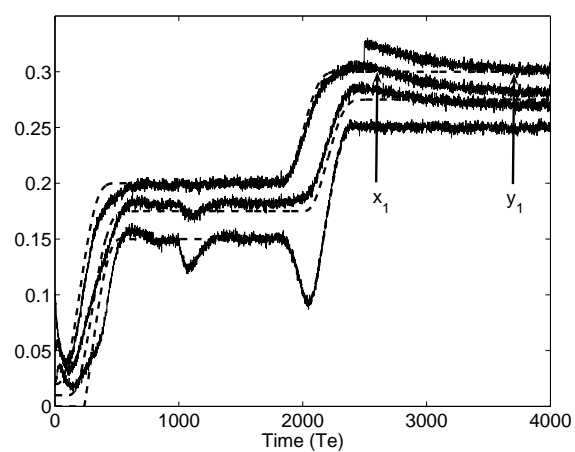

(a) Without fault-tolerant control

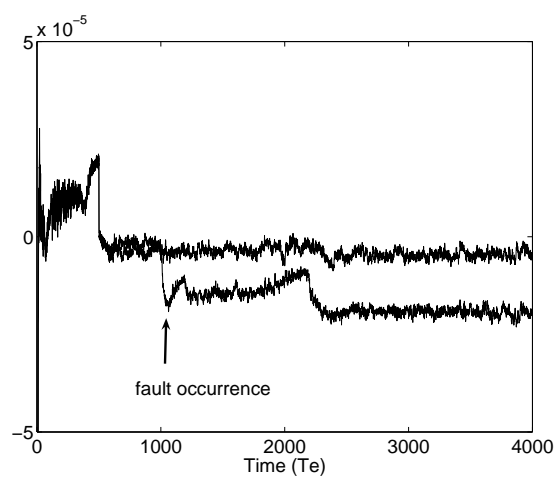

(c) $r_{2}$

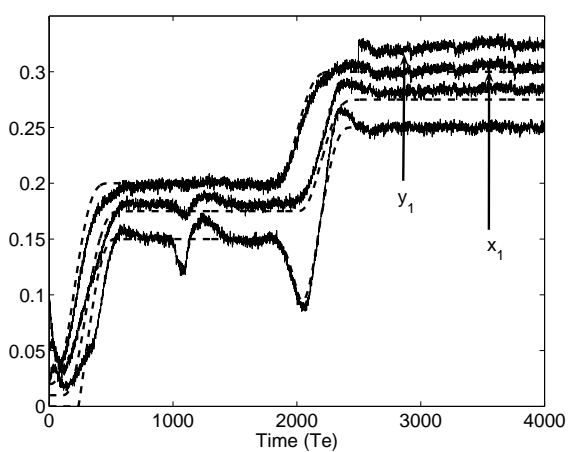

(b) With fault-tolerant control

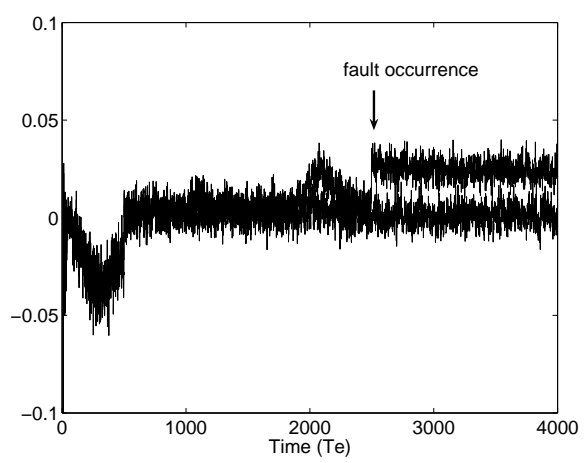

(d) $r_{4}$

Fig. 7. Leak and sensor faults

solutions of long-standing problems in nonlinear control are robust with respect to a large variety of perturbations and may be quite easily implemented in real time. They were made possible by a complete change of viewpoint on estimation techniques, where the classic asymptotic and/or probabilistic methods are abandoned ${ }^{11}$.

Further studies will demonstrate the possibility of controlling nonlinear systems with poorly known models, i.e., not only with uncertain parameters.

\section{References}

1. AMIRA-DTS2000, Laboratory setup three tank system. Amira Gmbh, Duisburg, 1996.

${ }^{11}$ See [16] for more details. 


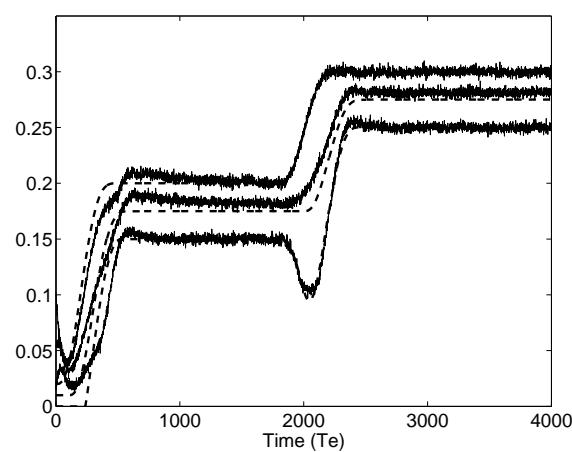

(a) Fault-free case

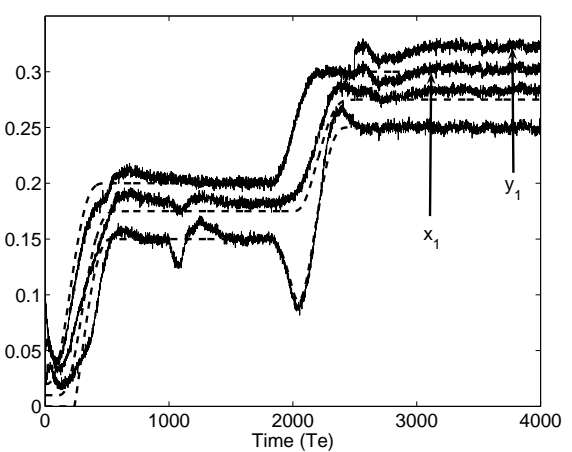

(b) Leak and sensor faults with fault-tolerant control

Fig. 8. Control feedback with the estimated viscosity coefficients

2. M. Basseville, V. Nikiforov, Detection of Abrupt Changes: Theory and Application. Prentice Hall, Englewood Cliffs, NJ, 1993.

3. M. Blanke, M. Kinnaert, J. Lunze, M. Staroswiecki, Diagnosis and Fault-Tolerant Control, Springer, Berlin, 2003.

4. A. Chambert-Loir, A Field Guide to Algebra, Springer, Berlin, 2005.

5. J. Chen, R. Patton, Robust Model-Based Fault Diagnosis for Dynamic Systems, Kluwer, Boston, 1999.

6. E. Delaleau, Algèbre différentielle, in Mathématiques pour les Systèmes Dynamiques, J.P. Richard (Ed.), vol. 2, chap. 6, pp. 245-268, Hermès, Paris, 2002.

7. S. Diop, M. Fliess, On nonlinear observability, Proc. $1^{\text {st }}$ Europ. Control Conf., Hermès, Paris, 1991, 152-157.

8. S. Diop, M. Fliess, Nonlinear observability, identifiability and persistent trajectories, Proc. $36^{\text {th }}$ IEEE Conf. Decision Control, Brighton, 1991, 714-719.

9. M. Fliess, C. Join, M. Mboup, H. Sira-Ramírez, Compression différentielle de transitoires bruités, C.R. Acad. Sci. Paris, série I, 339, 2004, 821-826.

10. M. Fliess, C. Join, H. Mounier, An introduction to nonlinear fault diagnosis with an application to a congested internet router, in Advances in Communication Control Networks, S. Tabouriech, C.T. Abdallah, J. Chiasson (Eds), Lect. Notes Control Inform. Sci., vol. 308, Springer, Berlin, 2005, pp. 327-343.

11. M. Fliess, C. Join, H. Sira-Ramírez, Robust residual generation for linear fault diagnosis: an algebraic setting with examples, Internat. J. Control, 77, 2004, 12231242 .

12. M. Fliess, J. Lévine, P. Martin, P. Rouchon, Flatness and defect of non-linear systems: introductory theory and examples, Internat. J. Control, 61, 1995, 13271361.

13. M. Fliess, J. Lévine, P. Martin, P. Rouchon, A Lie-Bäcklund approach to equivalence and flatness of nonlinear systems, IEEE Trans. Automat. Control, 44, 1999, 922-937.

14. M. Fliess, M. Mboup, H. Mounier, H. Sira-Ramírez, Questioning some paradigms of signal processing via concrete examples, in Algebraic Methods in Flat- 
ness, Signal Processing and State Estimation, H. Sira-Ramírez, G. Silva-Navarro (Eds.), Editiorial Lagares, México, 2003, pp. 1-21.

15. M. Fliess, H. Sira-Ramírez, An algebraic framework for linear identification, ESAIM Control Optim. Calc. Variat., 9, 2003, 151-168.

16. M. Fliess, H. Sira-Ramírez, Control via state estimations of some nonlinear systems, Proc. $6^{\text {th }}$ IFAC Symp. Nonlinear Control Syst. (NOLCOS), Stuttgart, 2004.

17. P.M. Frank, Fault diagnosis dynamic systems using analytical and knowledgebased redundancy - A survey and some new results, Automatica, 26, 1990, 459-474.

18. J. Gertler, Survey of model-based failure detection and isolation in complex plants, IEEE Control Systems Magazine, 8, 1988, 3-11.

19. J. Gertler, Fault Detection and Diagnosis in Engineering Systems, Marcel Dekker, New York, 1998.

20. V. Hagenmeyer, E. Delaleau, Exact feedforward linearization based on differential flatness, Internat. J. Control, 76, 2003, 537-556.

21. V. Hagenmeyer, E. Delaleau, Robustness analysis of exact feedforward linearization based on differential flatness, Automatica, 39, 2003, 1941-1946.

22. D. Henry, A. Zolghadri, M. Monsion, S. Ygorra, Off-line robust fault diagnosis using the generalized structured singular value, Automatica, 38, 2002, 1347-1358.

23. R. Isermann, P. Ballé, Trends in the application of model-based fault detection and diagnosis of technical processes, Control Eng. Practice, 5, 1997, 709-719.

24. A. Isidori, Nonlinear Control Systems, $3^{\text {rd }}$ ed., Springer, London, 1995.

25. C. Join, H. Sira-Ramírez, M. Fliess, Control of an uncertain three-tank-system via on-line parameter identification and fault detection, Proc. $16^{\text {th }}$ IFAC World Congress on Automatic Control, Prague, 2005.

26. E. Kolchin, Differential Algebra and Algebraic Groups, Academic Press, New York, 1973.

27. M. Krstić, I. Kanellakopoulos, P. Kokotović, Nonlinear and Adaptative Control Design, Wiley, New York, 1995.

28. S. Lang, Algebra, $3^{\text {rd }}$ ed., Addison-Wesley, Reading, MA, 1993.

29. J. Lunze, J. Askari-Marnani, A. Cela, P.M. Frank, A.L. Gehin, T. Marku, L. Rato, M. Staroswiecki, Three-tank control reconfiguration, in Control of Complex Systems, K.J. Aström, D. Blanke, A. Isidori, W. Schaufelberger, R. Sanz (Eds), Springer, Berlin, 2001, pp. 241-283.

30. R. Marino, P. Tomei, Nonlinear Control Design: Geometric, Adaptative and Robust, Prentice-Hall, London, 1995.

31. P. Martin, P. Rouchon, Systèmes plats de dimension finie, in Commandes non linéaires, F. Lamnabhi-Lagarrigue, P. Rouchon (Eds), Hermès, Paris, 2003.

32. P. Martin, P. Rouchon, Catalogue de systèmes plats, in Commandes non linéaires, F. Lamnabhi-Lagarrigue, P. Rouchon (Eds), Hermès, Paris, 2003.

33. J. Rudolph, Beiträge zur flacheitsbasierten Folgeregelung linearer und nichtlinearer Syteme endlicher und undendlicher Dimension, Shaker Verlag, Aachen, 2003.

34. L. Schwartz, Théorie des distributions, $2^{e}$ éd., Hermann, Paris, 1966.

35. H. Sira-Ramírez, S. Agrawal, Differentially Flat Systems, Marcel Dekker, New York, 2004.

36. H. Sira-Ramírez, M. Fliess, On the output feedback control of a synchronous generator, Proc. $43^{\text {rd }}$ IEEE Conf. Decision Control, Atlantis, Bahamas, 2004.

37. J.T. Spooner, M. Maggiore, R. Ordonez, K.M. Passino, Stable Adaptative Control and Estimation for Nonlinear Systems, Wiley, New York, 2002. 


\section{Index}

accomodation, 2

detectability, 4

differential algebra, 2

dynamics, 4

estimation of the time derivatives, 5

fault indicator, 8

fault variable, 3

fault-tolerant control, 2

flat hybrid system, 7

flat system, 4 identifiability, 5

input-output system, 4

isolability, 4

nonlinear system, 2

observability, 5

residual, 8

three-tank system, 6

uncertain parameters, 3 
